\title{
Is there any role of radiotherapy in intrahepatic cholangiocarcinomas?
}

\begin{abstract}
Cholangiocarcinoma or bile duct cancer is a rare type of cancer which is prevalent in developed countries and its prevalence is increasing in developing countries. Cholangiocarcinoma is included in the list of lethal cancer. While surgery is the cornerstone of its management, the benefit of adding neoadjuvant or adjuvant treatment in the form of chemotherapy and/or radiotherapy have not been shown to significantly improving the prognosis of the patients. The role of radiotherapy in the management of intrahepatic cholangiocarcinoma remains debated. These cancers usually present at advanced stages and are associated with low rates of local control and overall survival. The indications and implications of radiotherapy in these carcinomas are still not very clear. However, planning target volume and biologically effective dose of radiation have a prognostic value and initial treatment response is helpful in predicting survival time. In this review, we tried to explore various ways and options where radiotherapy can provide some better results in treating resectable and unresectable IHCC.
\end{abstract}

Volume 4 Issue 4 - 2017

\author{
Nupur Bansal,' Abhishek Soni² \\ 'Department of Radiotherapy, King George Medical University, \\ India \\ ${ }^{2}$ Department of Radiotherapy, Indraprastha Apollo Hospital, \\ India
}

Correspondence: Nupur Bansal, King George Medical University, Lucknow, Uttar Pradesh, India, Tel +918053442667, Email drnupurbansal@gmail.com

Received: May 14, 2017 | Published: November 02, 2017

Keywords: intrahepatic, cholangiocarcinoma, radiotherapy, adjuvant, neoadjuvant, palliative, proton

Abbreviations: IHCC, intrahepatic cholangiocarcinoma; EHCC, extrahepatic cholangiocarcinoma; CT, computed tomography; RFS, recurrence free survival

\section{Introduction}

Cholangiocarcinoma is a rare type of hepatobiliary cancer with 5year survival rate of $<5 \%$ and associated with high mortality rate. ${ }^{1}$ It is classified into intrahepatic cholangiocarcinoma (IHCC) and extrahepatic cholangiocarcinoma (EHCC). Hilar cholangiocarcinoma is most common $(60 \%)$ with distal $(30 \%)$ and IHCC $(10 \%)$ being less common. ${ }^{2}$ Due to the rarity of incidence of IHCC, there are no established treatment guidelines. Surgical resection remains the main treatment modality in the resectable cases. Role of radiotherapy in neoadjuvant, adjuvant and palliative settings remains uncertain. The addition of radiotherapy to chemotherapy is associated with improved survival in unresectable IHCC. ${ }^{3}$ With improvements in systemic therapy and radiation technique including the use of intensitymodulated and stereotactic body radiotherapy, radiotherapy may play an increasingly important role in improving survival.

\section{Discussion}

IHCC is a heterogeneous cancer type and complete surgical resection is the most effective treatment. Majority of the patients undergo postoperative adjuvant treatment in the form of chemotherapy or radiotherapy or combination of both the modalities. Although these adjuvant treatments have demonstrated certain benefit in survival rates and local control rates, the standard guidelines vary, especially regarding radiotherapy. ${ }^{3}$ At present, there is no consensus regarding the indications of radiotherapy, target contouring, dose and fractionation schedule of the radiotherapy treatment. Radiological modalities, including magnetic resonance cholangiopancreatography, dynamic computed tomography (CT), and positron emission tomography/CT have become useful for target contouring. Moreover, liver motion is a critical factor that requires consideration during contouring.
Fluoroscopy or 4D-CT is useful taking into consideration the liver motion and thus is helpful in the precise definition of the internal target volume. ${ }^{4-6}$ Tao et al have recently demonstrated that radiation dose impacts survival in patients with unresectable IHCC. ${ }^{7}$ Higher doses of radiation could have resulted in survival benefit. Also, in metastatic cases, radiotherapy did not confer survival benefit as these patients were more likely treated with palliative courses of radiotherapy. ${ }^{7}$

\section{Adjuvant therapy}

The benefit of adjuvant radiotherapy in patients with resectable IHCC remains controversial though certain factors might affect the treatment outcomes and survival of patients. ${ }^{1}$ Tumor histopathology, lymph node metastasis, positive margins, tumor stage are also prognostic factors influencing the outcome of the patient. ${ }^{8}$ Horgan et al., ${ }^{9}$ in a meta-analysis suggested that 5-FU based chemoradiation or chemotherapy is better to radiotherapy alone as adjuvant treatment especially in patients who underwent R1 resection or had lymph node positive disease. ${ }^{9}$ After R0 resection without any other risk factor, observation alone is appropriate. ${ }^{9}$ In another study by Shinohara et al, median OS significantly increases from 6months to 11 months when adjuvant radiation therapy is added to surgery. ${ }^{10}$

\section{Neoadjuvant therapy}

The role of neoadjuvant chemoradiotherapy is also controversial, though it might improve OS and recurrence free survival (RFS). Chemoradiation therapy regimen consisted of 3 cycles of full-dose gemcitabine $\left(1000 \mathrm{mg} / \mathrm{m}^{2}\right.$ at days 1,8 , and 15 , every 4 weeks) with 50-60Gy radiation. This was followed by surgery for IHCC or EHCC. In this study by Kobayashi et al., ${ }^{11}$ the three-year RFS rates in patients treated with and without neoadjuvant therapy were $78 \%$ and $58 \%$, respectively $(\mathrm{p}=0.0263) .{ }^{11}$

\section{Unresectable IHCC}

The prognosis of unresectable patients is poor with a median 
survival of 3.9 months with supportive care alone. ${ }^{12}$ Chemotherapy is an accepted standard of care for inoperable patients and the role of radiation is less well defined. Radiotherapy may improve bile duct patency and reduce pain in inoperable patients. ${ }^{2}$ Radiotherapy in a palliative setting has increased the patients' survival by 4 months in unresectable IHCC. In an another study by Chen et al, one-and twoyear survival rates for EBRT versus non-EBRT group were 38.5\% versus $16.4 \%$, and $9.6 \%$ versus $4.9 \%$, respectively. ${ }^{13}$ While there has been no randomized evidence for radiotherapy, a phase 2 study showed that radiation with concurrent hepatic artery oxuridine infusion was associated with improved survival over historical controls. ${ }^{14}$

\section{SBRT for IHCC}

Mahadevan et al. ${ }^{15}$ treated unresectable IHCC with SBRT and have given radiotherapy dose of $30 \mathrm{~Gy}$ in 3 fractions with some patients receiving chemotherapy also. After a median follow-up of 38 months, 1year local control and OS was $88 \%$ and $58 \%$, respectively. ${ }^{15}$ Jung et al. ${ }^{16}$ treated unresectable and recurrent cases of IHCC and EHCC with total doses of SBRT being given as $45 \mathrm{~Gy}$ in 3 fractions. ${ }^{16}$ Some patients have received EBRT prior to SBRT (median 40Gy in 20 fractions). One-year local control and median survival was $85 \%$ and 10months respectively. ${ }^{16}$

\section{Moderately hypofractionated proton therapy}

High dose hypofractionated proton therapy demonstrated better local control rate and overall survival in patients of IHCC. Proton therapy has also demonstrated better local control in IHCC and is especially useful in patients with underlying liver dysfunction. The CONSORT study has delivered 58GyE proton therapy in 15 fractions to unresctable cases of IHCC and hepatocellular carcinomas. ${ }^{17}$ Median follow up was of 19.5 months and 2 years local control and overall survival for IHCC was $94.1 \%$ and $46.5 \%$ respectively. ${ }^{17}$

\section{Conclusion}

To conclude, radiation in combination with chemotherapy results in prolonged overall survival in comparison to chemotherapy alone in patients with unresectable and/or advanced IHCC. SBRT and proton beam RT also have better local control and overall survival in patients ineligible for surgical resection. So, we strongly recommend radiation in the management of intrahepatic cholangiocarcinoma.

\section{Acknowledgements}

None.

\section{Conflict of interest}

Author declares that there is no conflict of interest.

\section{References}

1. Nantajit D, Trirussapanich P, Rojwatkarnjana S, et al. Clinical analysis of cholangiocarcinoma patients receiving adjuvant radiotherapy. $\mathrm{Mol}$ Clin Oncol. 2016;5(6):797-802.
2. Pollom E, Alagappan M, Park LS, et al. Does radiotherapy still have a role in unresected biliary tract cancer? Cancer Med. 2017;6(1):129-141.

3. Lischalk JW, Repka MC, Unger K. Radiation therapy for hepatobiliary malignancies. J Gastrointest Oncol. 2017;8(2):279-292.

4. Lin $\mathrm{G}$, Toh $\mathrm{CH}, \mathrm{Wu} \mathrm{RC}$, et al. Combined hepatocellular cholangiocarcinoma: prognostic factors investigated by computed tomography/magnetic resonance imaging. Int J Clin Pract. 2008;62(8):1199-1205.

5. Maccioni F, Martinelli M, Al Ansari N, et al. Magnetic resonance cholangiography: past, present and future: A review. Eur Rev Med Pharmacol Sci. 2010;14(8):721-725.

6. Breitenstein S, Apestegui C, Clavien PA. Positron emission tomography (PET) for cholangiocarcinoma. HPB (Oxford). 2008;10(2):120-121.

7. Tao R, Krishnan S, Bhosale PR, et al. Ablative radiotherapy doses lead to a substantial prolongation of survival in patients with inoperable intrahepatic cholangiocarcinoma: a retrospective dose response analysis. Journal of Clinical Oncology. 2016;34(3):219-226.

8. Chen MF. Peripheral cholangiocarcinoma (cholangiocellular carcinoma): Clinical features, diagnosis and treatment. J Gastroenterol Hepatol. 1999;14(2):1144-1149.

9. Horgan AM, Amir E, Walter T, et al. Adjuvant therapy in the treatment of biliary tract cancer: a systematic review and meta-analysis. J Clin Oncol. 2012;30(16):1934-1940.

10. Shinohara ET, Mitra N, Guo M, et al. Radiotherapy is associated with improved survival in adjuvant and palliative treatment of extrahepatic cholangiocarcinomas. Int J Radiat Oncol Biol Phys. 2009;74(4):1191-1198.

11. Kobayashi S, Tomokuni A, Gotoh $\mathrm{K}$, et al. A retrospective analysis of the clinical effects of neoadjuvant combination therapy with full-dose gemcitabine and radiation therapy in patients with biliary tract cancer. Eur J Surg Oncol. 2017;43(4):763-771.

12. Park J, Kim MH, Kim KP, et al. Natural history and prognostic factors of advanced cholangiocarcinoma without surgery, chemotherapy, or radiotherapy: a large-scale observational study. Gut Liver. 2009;3(4):298-305.

13. Chen YX, Zeng ZC, Tang ZY, et al. Determining the role of external beam radiotherapy in unresectable intrahepatic cholangiocarcinoma: A retrospective analysis of 84 patients. BMC Cancer. 2010;10:492.

14. Ben-Josef, Normolle ED, Ensminger WD, et al. Phase II trial of high-dose conformal radiation therapy with concurrent hepatic artery oxuridine for unresectable intrahepatic malignancies. J Clin Oncol. 2005;23(34):8739-8747.

15. Mahadevan A, Dagoglu N, Mancias J, et al. Stereotactic Body Radiotherapy (SBRT) for Intrahepatic and Hilar Cholangiocarcinoma. J Cancer. 2015;6(11):1099-1104.

16. Jung J, Yoon SM, Kim SY, et al. Radiation-induced liver disease after stereotactic body radiotherapy for small hepatocellular carcinoma: clinical and dose-volumetric parameters. Radiat Oncol. 2013;8:249.

17. Hong TS, Wo JY, Yeap BY, et al. Multi-Institutional Phase II Study of High-Dose Hypofractionated proton beam therapy in patients with localized, Unresectable Hepatocellular carcinoma and Intrahepatic Cholangiocarcinoma. J Clin Oncol. 2016;34(5):460-468. 